ALEA, Lat. Am. J. Probab. Math. Stat. 13, 521-544 (2016)

DOI: 10.30757/ALEA.v13-21

\title{
Self-avoiding walks on finite graphs of large girth
}

\author{
Ariel Yadin \\ Ben-Gurion University of the Negev \\ Dept. of Math., \\ PO Box 653, \\ Be'er Sheva, 8410501 ISRAEL. \\ E-mail address: yadina@bgu.ac.il \\ URL: https://www.math.bgu.ac.il/ yadina
}

\begin{abstract}
We consider self-avoiding walk on finite graphs with large girth. We study a few aspects of the model originally considered by Lawler, Schramm and Werner on finite balls in $\mathbb{Z}^{d}$. The expected length of a random self avoiding path is considered. We discuss possible definitions of "critical" behavior in the finite volume setting. We also define a "critical exponent" $\gamma$ for sequences of graphs of size tending to infinity, and show that $\gamma=1$ in the large girth case.
\end{abstract}

\section{Introduction}

1.1. Self-avoiding walks. A self-avoiding walk is a path in a graph that does not visit any vertex more than once. Counting the number of self-avoiding walks of length $n$ started at the origin in $\mathbb{Z}^{d}$, is a long standing open problem. It is very difficult to come up with formulas that capture the correct asymptotics. In fact, even the exponential growth rate of the number of such walks is difficult to precisely calculate in most cases; this number $\mu$ is known as the connective constant of the lattice. For more on self-avoiding walks in the Euclidean context see Bauerschmidt et al. (2012); Madras and Slade (2013).

Let us briefly introduce the main model usually considered in the plane (and in fact in $\mathbb{Z}^{d}$ in general). This is sometimes called Lawler-Schramm-Werner model of self-avoiding walk, see Lawler et al. (2004). For some parameter $0<x \in \mathbb{R}$ and scaling factor $\delta>0$ consider the finite graph $G_{\delta}:=\delta \mathbb{Z}^{d} \cap B(0,1)$, where $B(0,1)$ is the Euclidean ball of radius 1 . Let $\mathrm{SAW}_{\delta}$ be the set of all self-avoiding walks in $G_{\delta}$ started at 0 with an endpoint in the boundary of $G_{\delta}$ (there are finitely many such walks). We may define a probability measure $\mathbb{P}_{x}^{\delta}$ on $\mathrm{SAW}_{\delta}$ by letting the probability of $\omega \in \mathrm{SAW}_{\delta}$ be proportional to $x^{|\omega|}$ where $|\omega|$ is the length of $\omega$.

Received by the editors February 4, 2015; accepted June 16, 2016.

2010 Mathematics Subject Classification. 82B41, 60K35.

Key words and phrases. Self-avoiding walk, large girth graphs, critical exponents.

Supported by the Israel Science Foundation (grant no. 1346/15). 
It is known that the model undergoes a phase transition at $x_{c}=\mu^{-1}$. Ioffe (1998) has shown that for $x<x_{c}$ the measures $\mathbb{P}_{x}^{\delta}$ converge (in an appropriate sense) to a measure on geodesics from 0 to the boundary of $B(0,1)$. For $x>x_{c}$ one may show that the limiting curve fills the ball $B(0,1)$ (again, in an appropriate sense), see Duminil-Copin et al. (2014).

The major question is to understand what happens at the critical point $x=x_{c}$. In dimensions $d \geq 4$ the limiting curve is expected to be scaling to a Brownian motion; this is related to works of Brydges and Spencer (1985) and Hara (2008); Hara and Slade (1991, 1992b,a) (see also Brydges et al. (2012, 1992); Brydges and Imbrie (2003a,b); Brydges et al. (2009); Brydges and Slade (2010) for the upper critical dimension $d=4$, and the book Madras and Slade (2013) and references therein). This is known for $d \geq 5$ using lace expansion, see Hara and Slade (1992b). Dimension $d=3$ is the most mysterious. In dimension $d=2$ the limiting curve is conjectured to be $\mathrm{SLE}_{8 / 3}$, Schramm-Loewner Evolution of parameter $\frac{8}{3}$ Lawler et al. (2004). Not much has been rigorously proven regarding the critical twodimensional case; even very intuitive facts are quite involved, see e.g. Duminil-Copin and Hammond (2013).

1.2. Finite graphs. Self-avoiding walks on general graphs have received much less attention than the Euclidean lattices. (See, for example, Alm and Janson (1990); Grimmett and Li (2013); Madras and Wu (2005) and references therein.) In this note we adapt the Lawler-Schramm-Werner model to the setting of finite graphs. The problem on finite graphs is that there is no canonical way to define "criticality" and it is not clear what "mean field behavior" is. This is the purpose of our definition of the critical exponent $\gamma$ and critical sequences below, and their relations to the expected length of a self-avoiding path and the asymptotic behavior of the partition function. This is explained in analogy to the more classical Euclidean space setting.

There are two main results of this paper. The first, is the definition of the notion of critical sequences and critical exponent for the finite graph setting, with the different viewpoints relating them to expected length and intersection of independent self-avoiding paths. This is presented in Section 1.5 and Theorem 1.4.

The second main result is the analysis of the critical behavior of self-avoiding walks in the large-girth case. We have two different types of possible "mean field" behaviors (in analogy to the complete graph case and the Euclidean case), and we show that large girth graphs only exhibit one of these (namely critical exponent $\gamma=1$ ). This is done in Theorems 1.7 and 1.8.

Let us precisely define the model and state the results.

1.3. The model. A path $\omega$ in a graph $G$ is a sequence $\left(\omega_{0}, \omega_{1}, \ldots, \omega_{n}\right)$ of vertices such that $\omega_{j} \sim \omega_{j+1}$ for all $j<n$, where $x \sim y$ means $x, y$ are adjacent in the graph $G$. For such a path $\omega=\left(\omega_{0}, \omega_{1}, \ldots, \omega_{n}\right)$ we use $|\omega|=n$ to denote the length of the path, which is the number of edges traversed.

For a graph $G$ we denote by $\operatorname{SAW}(G)$ the set of finite length self avoiding walks in $G$; that is,

$$
\operatorname{SAW}(G)=\left\{\omega: \omega \text { is a finite path and } \forall k \neq j, \omega_{k} \neq \omega_{j}\right\} .
$$


We use $\operatorname{SAW}(o, G)$ where $o$ is a vertex in $G$ to denote the set of all self avoiding walks in $G$ starting at $o . \operatorname{SAW}_{k}(G)\left(\operatorname{resp} . \operatorname{SAW}_{k}(o, G)\right)$ denotes the set of those self avoiding walks in $\operatorname{SAW}(G)$ (resp. $\operatorname{SAW}(o, G)$ ) which have length $k$.

Definition 1.1. Let $G$ be a graph (finite or infinite). Let $o \in G$ be some vertex.

For a real parameter $x>0$ define the partition function

$$
Z_{o, G}(x):=\sum_{\omega \in \operatorname{SAW}(o, G)} x^{|\omega|}=\sum_{n=0}^{\infty}\left|\operatorname{SAW}_{n}(o, G)\right| \cdot x^{n} .
$$

(When $G$ is infinite this converges for $x<\mu^{-1}$ where $\mu=\mu(G)$ is the connective constant of $G$.) For any $x$ for which the partition function converges, define a probability measure on $\operatorname{SAW}(o, G)$ by

$$
\mathbb{P}_{x, o, G}[\omega]=\left(Z_{o, G}\right)^{-1} \cdot x^{|\omega|} .
$$

When $G$ is a transitive graph we will usually omit the root vertex $o$, since any vertex plays the same role; e.g. on a transitive graph $G$ by $\mathbb{P}_{x, G}$ we mean $\mathbb{P}_{x, o, G}$ for some $o$.

Expectation under $\mathbb{P}_{x, o, G}$ is denoted $\mathbb{E}_{x, o, G}$ (or $\mathbb{E}_{x, G}$ when $G$ is transitive).

We will be interested in two main quantities:

- The expected length of a random element under $\mathbb{P}_{x, o, G}$; that is

$$
L(x, o, G)=\mathbb{E}_{x, o, G}[|\omega|]=\left(Z_{o, G}(x)\right)^{-1} \cdot \sum_{n=0}^{\infty}\left|\operatorname{SAW}_{n}(o, G)\right| \cdot n \cdot x^{n} .
$$

- The probability that two independent samples from $\mathbb{P}_{x, o, G}$ intersect trivially; that is,

$$
I(x, o, G)=\left(\mathbb{P}_{x, o, G} \times \mathbb{P}_{x, o, G}\right)\left[\omega \cap \omega^{\prime}=\{o\}\right] .
$$

(Here $\omega, \omega^{\prime}$ are independent samples each with law $\mathbb{P}_{x, o, G}$.)

Of course the analysis of the partition function $Z_{o, G}(x)$ plays an important part. As usual, the vertex $o$ is omitted in the notation for transitive graphs.

1.4. Critical exponents. We start by a brief review of critical exponents in the classical case, as motivation for our definition of the critical exponent $\gamma$ below.

Let $G$ be some transitive infinite graph, and fix some origin $o \in G$. The classical literature on self-avoiding walks is interested in determining the existence and values of the so-called critical exponents. We will not go into all the details here, see Bauerschmidt et al. (2012) and Madras and Slade (2013) for more. One of these critical exponents is $\gamma$. It is defined as the number such that for some constant $A$,

$$
\left|\operatorname{SAW}_{n}(o, G)\right| \sim A \cdot \mu^{n} \cdot n^{\gamma-1} \quad \text { as } n \rightarrow \infty .
$$

(Here $f(x) \sim g(x)$ as $x \rightarrow a$ means that $\lim _{x \rightarrow a} f(x) / g(x)=1$ and the limit exists.)

For example, lace expansion methods show that when $G=\mathbb{Z}^{d}$ for $d \geq 5$ we have that the exponent $\gamma$ exists and $\gamma=1$, see Hara and Slade (1991, 1992b,a). This is what is called mean field behavior, because the analogous quantity for the simple random walk is also 1 in dimensions $d \geq 5$.

$\gamma$ also has a probabilistic interpretation. If it exists, then the probability that two independent uniformly chosen self-avoiding walks from $\operatorname{SAW}_{n}(o, G)$ intersect only at the origin is $\sim c n^{1-\gamma}$ as $n \rightarrow \infty$. 
It is possible to show that the exponent $\gamma$ exists if and only if for some constants $c, c^{\prime}$

$$
Z_{G}(x) \sim c(1-\mu \cdot x)^{-\gamma} \quad \text { and } \quad L(x, G) \sim c^{\prime}(1-\mu \cdot x)^{-1} \quad \text { as } x \nearrow \mu^{-1},
$$

(see e.g. Bauerschmidt et al., 2012).

If $\gamma$ exists then $\gamma=\lim _{x \nearrow \mu^{-1}} \frac{\log Z_{o, G}(x)}{\log L(x, o, G)}$. Thus, with this intuition in mind we define

$$
\gamma(x, o, G):=\frac{\log Z_{o, G}(x)}{\log (L(x, o, G)+1)} .
$$

In Proposition 1.9 we show that for transitive $G$,

$$
L(x, G)+1=I(x, G) \cdot Z_{G}(x) .
$$

Plugging in the above predictions we have that if the exponent $\gamma$ exists then

$$
I(x, G) \sim c(1-x \mu)^{\gamma-1} .
$$

Thus, mean field $\gamma=1$, implies that $I(x, G)$ is bounded away from 0 as $x \nearrow \mu^{-1}$. Non mean field exponent, $\gamma>1$, implies that this probability converges to 0 as $x \nearrow \mu^{-1}$.

These properties motivate our definition of the critical exponent in the finite case.

1.5. Criticality in the finite setting. Now let us return to the finite setting. Let $\left(G_{n}\right)_{n}$ be a sequence of finite graphs with $\left|G_{n}\right| \rightarrow \infty$, and $o_{n} \in G_{n}$ some root vertex.

Definition 1.2. For a sequence $\left(x_{n}\right)_{n}$ of positive real numbers:

- We say that sequence $\left(x_{n}\right)_{n}$ is super-critical (for SAW on $\left.\left(o_{n}, G_{n}\right)_{n}\right)$ if

$$
\liminf _{n \rightarrow \infty} Z_{o_{n}, G_{n}}\left(x_{n}\right)=\infty .
$$

- We say that the sequence $\left(x_{n}\right)_{n}$ is sub-critical if

$$
\limsup _{n \rightarrow \infty} Z_{O_{n}, G_{n}}\left(x_{n}\right)<\infty \text {. }
$$

- We say that the sequence $\left(x_{n}\right)_{n}$ is critical if for any $0<\varepsilon<1$ the sequence $\left(x_{n}(1+\varepsilon)\right)_{n}$ is super-critical and the sequence $\left(x_{n}(1-\varepsilon)\right)_{n}$ is sub-critical.

Critical sequences are unique in the following sense.

Proposition 1.3. Let $\left(x_{n}\right)_{n}$ be a critical sequences for $\left(o_{n}, G_{n}\right)$. Then $\left(y_{n}\right)_{n}$ is a critical sequence if and only if $\frac{x_{n}}{y_{n}} \rightarrow 1$ as $n \rightarrow \infty$.

Another result is a characterization of critical sequences in terms of the intersection probability function $I$.

Theorem 1.4. For SAW on a sequence of transitive graphs $G_{n}$ with $\left|G_{n}\right| \nearrow \infty$, and a sequence $\left(x_{n}\right)_{n}$ :

- $\left(x_{n}\right)_{n}$ is super-critical if and only if $\lim \sup _{n} I\left(x_{n}, G_{n}\right)=0$.

- $\left(x_{n}\right)_{n}$ is sub-critical if and only if $\liminf _{n} I\left(x_{n}, G_{n}\right)>0$.

This theorem is not surprising, since we expect that super-critical paths should be "long", so that a trivial intersection has small probability, and sub-critical paths are expected to be short, so there is a reasonable probability of a trivial intersection. 
1.6. Mean field. The main purpose of this paper is to analyze the finite graph model. We begin with the simplest model, the complete graph.

Theorem 1.5. Consider the SAW model on the sequence $\left(G_{n}\right)_{n}$ where $G_{n}$ is the complete graph on $n$ vertices. Then, $\left(\frac{1}{n}\right)_{n}$ is a critical sequence.

Moreover, for all $\varepsilon>0$ :

- If $\left(x_{n}\right)_{n}$ is a sequence such that $\lim _{n \rightarrow \infty}\left(n x_{n}\right)=1+\varepsilon$, then

$$
\lim _{n \rightarrow \infty} \frac{L\left(x_{n}, G_{n}\right)}{n}=\frac{\varepsilon}{1+\varepsilon} \quad \text { and } \quad \lim _{n \rightarrow \infty} I\left(x_{n}, G_{n}\right)=0 .
$$

- If $\left(x_{n}\right)_{n}$ is a sequence such that $\lim _{n \rightarrow \infty}\left(n x_{n}\right)=1-\varepsilon$ then,

$$
\lim _{n \rightarrow \infty} L\left(x_{n}, G_{n}\right)=\frac{1-\varepsilon}{\varepsilon} \quad \text { and } \quad \lim _{n \rightarrow \infty} I\left(x_{n}, G_{n}\right)=1 .
$$

- If $x_{n}=\frac{1}{n}$ then there exists some (explicit) universal constant $\alpha>0$ such that

$$
\lim _{n \rightarrow \infty} \frac{L\left(x_{n}, G_{n}\right)}{\sqrt{n}}=\alpha .
$$

Theorem 1.6. Consider the SAW model on the sequence $\left(G_{n}\right)_{n}$ where $G_{n}$ is the complete graph on $n$ vertices. For critical and sub-critical sequences $\left(x_{n}\right)_{n}$ such that $\lim \sup _{n \rightarrow \infty}\left(n x_{n}\right) \leq 1$, the exponent $\gamma$ has the mean field value

$$
\lim _{n \rightarrow \infty} \gamma\left(x_{n}, G_{n}\right)=1 \text {. }
$$

For a super-critical sequence $\left(n x_{n}\right) \rightarrow 1+\varepsilon$, we have

$$
\lim _{n \rightarrow \infty} \gamma\left(x_{n}, G_{n}\right)=\infty \text {. }
$$

1.7. Euclidean case. It is interesting to compare our findings on the complete graph to the more classical Euclidean case.

When $G_{n}$ is the $n \times n$ torus $(\mathbb{Z} / n \mathbb{Z})^{2}$, the above remarks on the planar selfavoiding walk lead us to the conclusion that

$$
L\left(x, G_{n}\right)= \begin{cases}O(1) & x<\mu\left(\mathbb{Z}^{2}\right)^{-1} \\ \Omega\left(n^{2}\right) & x>\mu\left(\mathbb{Z}^{2}\right)^{-1} \\ n^{4 / 3} & x=\mu\left(\mathbb{Z}^{2}\right)^{-1},\end{cases}
$$

where the last value in the critical case is only conjectured, and has to do with the fact the the Hausdorff dimension of $\mathrm{SLE}_{\kappa}$ is $1+\frac{\kappa}{8}$ for $\kappa \leq 8$. (Note that now walks are not constrained to reach the boundary of the ball of radius $n$, as would be the case in the original Lawler-Schramm-Werner model.)

For higher dimensions (that is, $\left.(\mathbb{Z} / n \mathbb{Z})^{d}\right)$ the sub-critical and super-critical behavior should be the same as the planar case; i.e. constant for sub-critical and order of the volume in the super-critical case. The results of Brydges and Spencer (1985) and Hara and Slade (1991, 1992b,a) tell us that the the number of selfavoiding walks in $\mathbb{Z}^{d}, d \geq 5$, started at 0 , of length $n$, is asymptotic to $A \mu^{n}$, for some constant $A>0$, and that a typical self-avoiding walk of length $n$ behaves like a random walk. This leads us to expect that on the finite torus $(\mathbb{Z} / n \mathbb{Z})^{d}, d \geq 5$ the self-avoiding walk should not "feel" its own presence until reaching length $n^{d / 2}$ (this is just the birthday paradox). Thus, we expect that for $d \geq 5$ taking $G_{n}=(\mathbb{Z} / n \mathbb{Z})^{d}$, we have that

$$
L\left(x_{c}, G_{n}\right)=\Theta\left(n^{d / 2}\right)=\sqrt{\left|G_{n}\right|} .
$$


Here $x_{c}$ is the critical parameter $x_{c}=\mu\left(\mathbb{Z}^{d}\right)^{-1}$.

The complete graph together with the Euclidean case give two possible notions of "mean field behavior": critical exponent $\gamma=1$ or expected length $L$ of order square-root of the volume. We turn to the large girth case to understand which of these is the correct picture when there is more exotic geometry involved.

1.8. SAW on large girth. Expander graphs are graphs with very good expansion properties. It is known that a random $d$-regular graph is a very good expander with high probability, and has large girth around typical vertices with high probability. In many models random $d$-regular graphs exhibit the same behavior as the meanfield complete graph case. Sometimes such mean-field behavior can also be shown for expanders of large girth in general. See the discussion immediately following Theorem 1.7.

This experience may lead one to conjecture that the same phenomena will occur for the SAW model. The sub-critical and super-critical cases indeed do have constant and linear order expected length respectively. However, the analogy breaks down for the expected length $L$ in the critical case, and the scaling is not $\sqrt{\left|G_{n}\right|}$. That being said, a different measure of "mean field" is the critical exponent $\gamma$. In this case we will show that $\gamma=1$ for graphs of large girth.

Instrumental in the proofs are non-backtracking random walks. A non-backtracking walk is a path in a graph that never backtracks the last edge it passed through. A non-backtracking random walk is one that chooses each step randomly out of the currently allowed steps. This is a Markov chain on the set of directed edges of the graph. For a non-backtracking random walk on a graph $G$ one may define the mixing time by

$$
\tau=\min \left\{t: \max _{u, v}\left|\mathbb{P}_{u}[\mathrm{NB}(t)=v]-\frac{1}{|G|}\right| \leq \frac{1}{2|G|}\right\},
$$

where NB is the non-backtracking random walk, and $\mathbb{P}_{v}$ is the associated probability measure conditioned on $\mathrm{NB}(0)=v$. In Alon et al. (2007) the mixing time of nonbacktracking random walks is studied, and it is shown that it is always better than the mixing time of the usual simple random walk. We use the mixing time of the non-backtracking random walk to quantitatively define the notion of "large girth".

Theorem 1.7. Let $\left(G_{n}\right)_{n}$ be a sequence of $d$-regular transitive graphs with sizes $\left|G_{n}\right| \nearrow \infty$. Let $g_{n}$ be the girth of $G_{n}$ and assume that $g_{n} \rightarrow \infty$ as $n \rightarrow \infty$. Consider the SAW model on the sequence $\left(G_{n}\right)_{n}$.

Then, $\frac{1}{d-1}$ is a critical (constant) sequence, and we have:

- If $x_{n} \rightarrow x$ for $x<(d-1)^{-1}$ then

$$
\lim _{n \rightarrow \infty} L\left(x_{n}, G_{n}\right)=\frac{(d-1) x}{1-(d-1) x} \quad \text { and } \quad \lim _{n \rightarrow \infty} I\left(x_{n}, G_{n}\right)=1-\frac{1}{d} \text {. }
$$

- If $x=(d-1)^{-1}$ then,

$$
C^{-1} g_{n} \leq L\left(x, G_{n}\right) \leq C \cdot g_{n}(d-1)^{2 g_{n}}
$$

where $C>0$ is a constant that depends only on the degree $d$.

- Assume that $G_{n}$ has large girth in the sense that if $\tau_{n}$ is the mixing time of the non-backtracking random walk on $G_{n}$, then $\tau_{n}=o\left((d-1)^{g_{n} / 4}\right)$ as $n \rightarrow \infty$. 


$$
\begin{aligned}
& \text { If } x_{n} \rightarrow x>(d-1)^{-1} \text { then there exists } c=c(x, d)>0 \text { such that } \\
& \qquad \liminf _{n \rightarrow \infty} \frac{L\left(x_{n}, G_{n}\right)}{\left|G_{n}\right|} \geq c \quad \text { and } \quad \lim _{n \rightarrow \infty} I\left(x_{n}, G_{n}\right)=0 .
\end{aligned}
$$

Note that one may find expander graphs $\left(G_{n}\right)_{n}$ with girth as large as $g_{n}=$ $\varepsilon \log \left|G_{n}\right|$ for any $\varepsilon>0$. By results of Alon et al. (2007), the mixing time of the non-backtracking random walk is faster than the mixing time of the simple random walk on $G_{n}$. Choosing $\left(G_{n}\right)_{n}$ as these large-girth expander graphs, the mixing time in this case is $\tau_{n}=O\left(\log \left|G_{n}\right|\right)$. So in Theorem 1.7 the critical expected length could be bounded by order $\left|G_{n}\right|^{\delta}$ for any choice of $\delta>0$ (and in fact may even be poly-logarithmic). For example, it is well known that the above conditions hold for the constructions by Margulis Margulis (1982) and the Lubotzky-Phillips-Sarnak expanders Lubotzky et al. (1988).

Occasionally one expects expanders, especially large girth expanders, to exhibit tree-like or mean-field behavior. To illustrate this, let us contrast our results with some other mean-field behavior in the large girth setting. In Nachmias (2009) finite graphs with large girth are shown to exhibit mean-field behavior of the critical window for bond percolation. Coincidently, the non-backtracking random walk appears as part of the conditions in that paper as well. A more recent paper Nachmias and Peres (2012) deals with the infinitely many infinite components phase in percolation on infinite graphs with large girth, as well as the critical exponents for percolation and self-avoiding walks on such graphs. There it is shown that the exponents are mean-field exponents for large girth infinite graphs. Again, there is a connection to the non-backtracking random walk, which appears in the proofs.

1.9. Critical exponent in large girth. As mentioned above, although the expected length $L$ does not exhibit "mean field behavior" for large girth expanders, we may consider another measure of "mean field behavior", namely the "critical exponent" $\gamma$. We show that $\gamma=1$ in the critical regime for graphs with large girth. We also determine $\gamma$ for the sub- and super-critical regimes.

Theorem 1.8. For a sequence of d-regular transitive graphs $\left(G_{n}\right)_{n}$, of size $\left|G_{n}\right| \nearrow$ $\infty$ and girth $g_{n} \rightarrow \infty$ as $n \rightarrow \infty$ :

- For the critical sequence $x_{n}=\frac{1}{d-1}$, the critical exponent $\gamma$ has the mean field value

$$
\lim _{n \rightarrow \infty} \gamma\left(x_{n}, G_{n}\right)=1
$$

- If $x_{n} \rightarrow x<\frac{1}{d-1}$ then

$$
\lim _{n \rightarrow \infty} \gamma\left(x_{n}, G_{n}\right)=\frac{\log \frac{d}{d-1}-\log (1-(d-1) x)}{-\log (1-(d-1) x)} \in(1, \infty) .
$$

- If $x_{n} \rightarrow x>\frac{1}{d-1}$ and if the girth of $G_{n}$ is large enough so that $\tau_{n}=o((d-$ $1)^{g_{n} / 4}$ ) as $n \rightarrow \infty$ (where $\tau_{n}$ is the mining time of the non-backtracking random walk on $G_{n}$, as in the assumptions of Theorem 1.7), then

$$
\lim _{n \rightarrow \infty} \gamma\left(x_{n}, G_{n}\right)=\infty \text {. }
$$

Recall that in the classical setting $I(x, G) \sim c(1-x \mu)^{\gamma-1}$ (as in Section 1.4). In our (large girth) setting the analogous quantity for a sequence $x_{n} \rightarrow x<\frac{1}{d-1}$ is

$$
\left(1-(d-1) x_{n}\right)^{\gamma\left(x_{n}, G_{n}\right)-1}=\exp \left(\log \frac{d}{d-1} \cdot \frac{\log \left(1-(d-1) x_{n}\right)}{-\log (1-(d-1) x)}\right) \rightarrow \frac{d-1}{d}>0 .
$$


Also, by Theorem 1.7, $I\left(x_{n}, G_{n}\right) \rightarrow \frac{d-1}{d}$. This coincides nicely with the fact that $\gamma=1$ in the critical case.

1.10. Some more general results. Our analysis also provides some general results relating the trivial-intersection probability $I(x, o, G)$ to the expected length $L(x, o, G)$ and partition function $Z_{o, G}(x)$, for transitive graphs. This relation also holds for infinite graphs (as long as the partition function converges).

Proposition 1.9. Suppose that $G$ is a transitive graph (finite or infinite). Let $x>0$ be such that $Z_{G}(x)$ converges (all $x>0$ in the finite graph case). Then,

$$
L(x, G)+1=I(x, G) \cdot Z_{G}(x) .
$$

We also prove that the expected length $L$ is an increasing function of the parameter. This result also holds for infinite graphs as long as the model is defined.

Theorem 1.10. The function $x \mapsto L(x, o, G)$ is a non-decreasing function (defined for all $x$ such that $\left.Z_{o, G}(x)<\infty\right)$.

\section{Open questions}

Before moving to the proofs, let us mention some basic open questions.

2.1. Critical exponents. There are other critical exponents which appear in the classical self-avoiding walk literature (e.g. mean-square displacement exponent $\nu$ ). It would be of interest to define these values in the finite graph setting, and perhaps obtain some relations similar to the Fischer relations (see e.g. Bauerschmidt et al., 2012).

Regarding the exponent $\gamma$, note that a-priori its value depends on the choice of the sequence of parameters $x_{n}$.

Question 2.1. Show that for two critical sequences $\frac{x_{n}}{y_{n}} \rightarrow 1$, we have that

$$
\frac{\gamma\left(x_{n}, o_{n}, G_{n}\right)}{\gamma\left(y_{n}, o_{n}, G_{n}\right)} \rightarrow 1
$$

Another phenomena arising from this work is that we expect $\gamma=\infty$ in the super-critical regime. This regime has no analogue in the infinite-graph case.

Question 2.2. Show that if $\left(x_{n}\right)_{n}$ is a super-critical sequence then $\gamma\left(x_{n}, o_{n}, G_{n}\right) \rightarrow$ $\infty$.

2.2. I is decreasing. The expected length of a self-avoiding walk $L(x, o, G)$ is an increasing function of $x$. Intuitively, raising $x$ increases the length of a typical path. We would expect that this phenomena should imply that two independent self-avoiding walks should have smaller chances of a trivial intersection as $x$ grows.

Question 2.3. Show that the function $x \mapsto I(x, o, G)$ is a non-increasing function of $x$. 
2.3. Extending to other models. In this paper we have dealt with transitive large girth graphs. Transitivity is required to relate the expected length $L$ to the intersection probability $I$, as in Proposition 1.9, which is a starting point for our analysis. The large girth is required for the connection to the non-backtracking walk, see Section 3 below.

It is very natural to extend the results in this paper and investigate graphs that are not necessarily transitive, and graphs that may have small cycles, but only very few. For example, this is what random $d$-regular graphs look like. It seems that the analysis becomes more difficult in these cases, and perhaps new ideas are required.

\section{Connection to non-backtracking random walk}

The connection between self-avoiding walks and non-backtracking walks has already appeared in a paper by Kesten (1964). It is used to give bounds on the connective constant of $\mathbb{Z}^{d}$ for large $d$.

It seems, in fact, that there is a deeper connection between self-avoiding walks and non-backtracking random walks. The theory is developed in Fitzner (2013), where Fitzner and van der Hofstad develop lace expansion based on the nonbacktracking random walk rather than the simple random walk. They apply this in $\mathbb{Z}^{d}$ to the analysis of SAW. However, it seems that lace expansion methods, while achieving amazing results in $\mathbb{Z}^{d}$, are difficult to generalize to other settings (such as non-commutative groups and even more general graphs).

We now give a simple application of non-backtracking random walks to the study of self avoiding walks.

A non-backtracking path $\omega$ in $G$ is a sequence $\omega=\left(\omega_{0}, \ldots, \omega_{n}\right)$ such that for all $j<n$ we have $\omega_{j} \sim \omega_{j+1}$ (so that $\omega$ is a path in $G$ ), and this path never backtracks an edge in $G$ : i.e. for all $j<n-1$ we have that $\omega_{j+2} \neq \omega_{j}$. We use NB to denote a non-backtracking random walk on a fixed $d$-regular graph $G$ started at a fixed vertex $o \in G$. $\mathbf{P}_{o}, \mathbf{E}_{o}$ denote the corresponding probability measure and expectation. Thus, for any non-backtracking path $\omega$ in $G$ of length $|\omega|=k$ started at $o$, the $\mathbf{P}_{o}$-probability that $\mathrm{NB}[0, k]=\omega$ is $\frac{1}{d} \cdot(d-1)^{-(k-1)}$. It is simple to see that this is obtained by the Markov chain on directed edges, that at each step chooses uniformly among all edges emanating from the current vertex, except the edge that was used to reach the current vertex in the previous time step.

The key observation is as follows.

$$
\left|\operatorname{SAW}_{k}(o, G)\right| \cdot\left(d(d-1)^{k-1}\right)^{-1}=\sum_{\omega \in \operatorname{SAW}_{k}} \frac{1}{d(d-1)^{k-1}}=\mathbf{P}_{o}[\mathrm{NB}[0, k] \in \operatorname{SAW}(o, G)]
$$

Define

$$
T=\inf \{k \geq 0: \operatorname{NB}[0, k] \notin \operatorname{SAW}(G)\}=\inf \{k>2: \exists j<k, \operatorname{NB}(j)=\mathrm{NB}(k)\}
$$

We call $T$ the self-intersection time of NB. Thus,

$$
\left|\operatorname{SAW}_{k}(o, G)\right|=\frac{d}{d-1} \cdot(d-1)^{k} \cdot \mathbf{P}_{o}[T>k]
$$


Now consider the partition function for any $0<y \neq 1$ and $x=\frac{y}{d-1}$,

$$
\begin{aligned}
Z_{o, G}(x) & =\sum_{k=0}^{\infty}\left|\operatorname{SAW}_{k}(o, G)\right|(d-1)^{-k} y^{k} \\
& =\frac{d}{d-1} \cdot \sum_{k=0}^{\infty} \mathbf{P}_{o}[T>k] y^{k}=\frac{d}{d-1} \cdot \frac{1}{y-1} \cdot \mathbf{E}_{o}\left[y^{T}-1\right]
\end{aligned}
$$

Since,

$$
\begin{aligned}
\sum_{y=0}^{m-1} k y^{k} & =y \frac{\partial}{\partial y} \sum_{k=0}^{m-1} y^{k}=y \frac{\partial}{\partial y} \frac{y^{m}-1}{y-1} \\
& =y \frac{m y^{m-1}(y-1)-y^{m}+1}{(y-1)^{2}}=\frac{y}{y-1} \cdot m y^{m-1}-\frac{y}{y-1} \cdot \frac{y^{m}-1}{y-1}
\end{aligned}
$$

we get that

$$
\begin{aligned}
\mathbb{E}_{x, o, G}[|\omega|] & =\left(Z_{o, G}(x)\right)^{-1} \cdot \frac{d}{d-1} \cdot \mathbf{E}_{o}\left[\sum_{k=0}^{\infty} k y^{k} \mathbf{1}_{\{T>k\}}\right] \\
& =\frac{\mathbf{E}_{o}\left[T y^{T}\right]}{\mathbf{E}_{o}\left[y^{T}-1\right]}-\frac{y}{y-1}=\frac{y}{1-y}-\frac{\mathbf{E}_{o}\left[T y^{T}\right]}{\mathbf{E}_{o}\left[1-y^{T}\right]}
\end{aligned}
$$

For the case $x=\frac{1}{d-1}($ i.e. $y=1)$,

$$
\begin{aligned}
Z_{o, G}(x) & =\sum_{k=0}^{n-1}\left|\operatorname{SAW}_{k}(o, G)\right|(d-1)^{-k} \\
& =\frac{d}{d-1} \cdot \sum_{k=0}^{n-1} \mathbf{P}_{o}[T>k]=\frac{d}{d-1} \cdot \mathbf{E}_{o}[T] .
\end{aligned}
$$

And similarly to the above,

$$
\mathbb{E}_{x, o, G}[|\omega|]=\left(Z_{o, G}(x)\right)^{-1} \cdot \frac{d}{d-1} \cdot \mathbf{E}_{o}\left[\sum_{k=0}^{\infty} k \mathbf{1}_{\{T>k\}}\right]=\frac{\mathbf{E}_{o}[T(T-1)]}{2 \mathbf{E}_{o}[T]}
$$

\section{Proofs of General Results}

We start with the proof of Proposition 1.3, which is the statement the critical sequences are unique (in a certain sense).

Proof of Proposition 1.3: To simplify the notation in this proof, we write $Z\left(x_{n}\right)$ for $Z_{o_{n}, G_{n}}\left(x_{n}\right)$.

For the first direction, assume that both $\left(x_{n}\right)_{n},\left(y_{n}\right)_{n}$ are critical sequences. It suffices to prove that $\lim \sup _{n \rightarrow \infty} \frac{x_{n}}{y_{n}} \leq 1$, since both sequences play the same role.

If $\limsup _{n \rightarrow \infty} \frac{x_{n}}{y_{n}}>1$, then there exists some $0<\varepsilon<\frac{1}{4}$ and subsequences $\left(x_{n_{k}}\right)_{k},\left(y_{n_{k}}\right)_{k}$ such that for all $k$,

$$
\frac{x_{n_{k}}}{y_{n_{k}}} \geq 1+4 \varepsilon \geq 1+\frac{2 \varepsilon}{1-\varepsilon}=\frac{1+\varepsilon}{1-\varepsilon} .
$$


Thus, by the definition of a critical sequence, using the fact that $Z_{o, G}(\cdot)$ is an increasing function,

$$
\begin{aligned}
\infty & >\limsup _{n \rightarrow \infty} Z\left(x_{n}(1-\varepsilon)\right) \geq \limsup _{k \rightarrow \infty} Z\left(x_{n_{k}}(1-\varepsilon)\right) \\
& \geq \limsup _{k \rightarrow \infty} Z\left(y_{n_{k}}(1+\varepsilon)\right) \geq \liminf _{n \rightarrow \infty} Z\left(y_{n}(1+\varepsilon)\right)=\infty,
\end{aligned}
$$

a contradiction!

For the other direction, let $\left(x_{n}\right)_{n}$ be a critical sequence and $\left(y_{n}\right)_{n}$ a sequence such that $\frac{x_{n}}{y_{n}} \rightarrow 1$.

Fix small $\varepsilon>0$. Then, for all large enough $n$ we have $x_{n}\left(1-\frac{1}{2} \varepsilon\right) \leq y_{n} \leq(1+\varepsilon) x_{n}$. Thus,

$$
\begin{aligned}
& \limsup _{n \rightarrow \infty} Z\left(y_{n}(1-\varepsilon)\right) \leq \limsup _{n \rightarrow \infty} Z\left(x_{n}\left(1-\varepsilon^{2}\right)\right)<\infty, \\
& \liminf _{n \rightarrow \infty} Z\left(y_{n}(1+\varepsilon)\right) \geq \liminf _{n \rightarrow \infty} Z\left(x_{n}\left(1+\frac{1}{2} \varepsilon(1-\varepsilon)\right)\right)=\infty .
\end{aligned}
$$

So $\left(y_{n}\right)_{n}$ is critical as well.

We move to the proof of Proposition 1.9, which is the identity $I Z=L+1$.

Proof of Proposition 1.9: Let

$$
S_{k, j}:=\left\{\left(\omega, \omega^{\prime}\right) \in \operatorname{SAW}_{k}(o, G) \times \operatorname{SAW}_{j}(o, G): \omega \cap \omega^{\prime}=\{o\}\right\} .
$$

For $\left(\omega, \omega^{\prime}\right) \in S_{k, j}$ let $\hat{\omega}$ be the path that is $\varphi\left(\omega \cup \omega^{\prime}\right)$ mapped by the automorphism $\varphi$ of $G$ taking $\omega(k)$ to $o$. Note that $\hat{\omega} \in \operatorname{SAW}_{k+j}(o, G)$ because $\omega \cap \omega^{\prime}=\{o\}$. This map may be inverted. If $\hat{\omega} \in \operatorname{SAW}_{k+j}(o, G)$ one can define a pair

$$
\omega=\varphi^{-1}(\hat{\omega}(k), \hat{\omega}(k-1), \ldots, \hat{\omega}(0)) \quad \text { and } \quad \omega^{\prime}=\varphi^{-1}(\hat{\omega}[k, k+j]),
$$

where $\varphi$ is the same automorphism as before. Then $\left(\omega, \omega^{\prime}\right) \in S_{k, j}$. Thus, we have shown that when $G$ is transitive, $\left|S_{k, j}\right|=\left|\mathrm{SAW}_{k+j}\right|$.

We now compute for $x=\frac{y}{d-1}, y \neq 1$,

$$
\begin{aligned}
& \left(Z_{o, G}(x)\right)^{2} \cdot \mathbb{P}\left[\omega \cap \omega^{\prime}=\{o\}\right]=\frac{d}{d-1} \cdot \sum_{k, j=0}^{n-1} y^{k+j} \mathbf{P}_{o}[T>k+j]=\frac{d}{d-1} \cdot \mathbf{E}_{o} \sum_{k=0}^{T-1} y^{k} \sum_{j=0}^{T-1-k} y^{j} \\
& =\frac{d}{d-1} \cdot \frac{1}{y-1} \cdot \mathbf{E}_{o} \sum_{k=0}^{T-1} y^{k}\left(y^{T-k}-1\right)=\frac{d}{d-1} \cdot \frac{1}{(y-1)^{2}} \cdot \mathbf{E}_{o}\left[T y^{T}(y-1)-y^{T}+1\right] .
\end{aligned}
$$

Thus,

$$
\mathbb{P}\left[\omega \cap \omega^{\prime}=\{o\}\right]=\frac{d-1}{d} \cdot \frac{\mathbf{E}_{o}\left[T y^{T}(y-1)-y^{T}+1\right]}{\left(\mathbf{E}_{o}\left[y^{T}-1\right]\right)^{2}} .
$$

So by (3.3),

$$
\begin{aligned}
\mathbb{E}_{x, o, G}[|\omega|]+1 & =\frac{\mathbf{E}_{o}\left[T y^{T}\right]}{\mathbf{E}_{o}\left[y^{T}-1\right]}-\frac{1}{y-1}=\frac{1}{y-1} \cdot \frac{\mathbf{E}_{o}\left[T y^{T}(y-1)-y^{T}+1\right]}{\mathbf{E}_{o}\left[y^{T}-1\right]} \\
& =\frac{1}{y-1} \cdot \frac{d}{d-1} \cdot \mathbb{P}\left[\omega \cap \omega^{\prime}=\{o\}\right] \cdot \mathbf{E}_{o}\left[y^{T}-1\right] \\
& =\mathbb{P}\left[\omega \cap \omega^{\prime}=\{o\}\right] \cdot Z_{o, G}(x) .
\end{aligned}
$$


In the case $x=\frac{1}{d-1}(y=1)$,

$$
\begin{aligned}
\left(Z_{o, G}(x)\right)^{2} \cdot \mathbb{P}\left[\omega \cap \omega^{\prime}=\{o\}\right] & =\frac{d}{d-1} \cdot \sum_{k, j=0}^{n-1} \mathbf{P}_{o}[T>k+j]=\frac{d}{d-1} \cdot \mathbf{E}_{o} \sum_{k=0}^{T-1}(T-k) \\
& =\frac{d}{d-1} \cdot \mathbf{E}_{o} \sum_{k=1}^{T} k=\frac{d}{d-1} \cdot \frac{1}{2} \mathbf{E}_{o}[T(T+1)] .
\end{aligned}
$$

So,

$$
\mathbb{P}\left[\omega \cap \omega^{\prime}=\{o\}\right]=\frac{d-1}{d} \cdot \frac{\mathbf{E}_{o}[T(T+1)]}{2\left(\mathbf{E}_{o}[T]\right)^{2}},
$$

and by (3.5),

$$
\begin{aligned}
\mathbb{E}_{x, o, G}[|\omega|]+1 & =\frac{\mathbf{E}_{o}[T(T+1)]}{2 \mathbf{E}_{o}[T]}=\frac{d}{d-1} \cdot \mathbb{P}\left[\omega \cap \omega^{\prime}=\emptyset\right] \cdot \mathbf{E}_{o}[T] \\
& =\mathbb{P}\left[\omega \cap \omega^{\prime}=\{o\}\right] \cdot Z_{o, G}(x) .
\end{aligned}
$$

We now prove Theorem 1.10, showing that $L(x)$ is an increasing function of $x$, regardless of the graph chosen (as long as it is defined).

Proof of Theorem 1.10: Define $P_{m}(x)=\mathbb{P}_{x, o, G}[|\omega|<m]$. We claim that $P_{m}$ is decreasing in $x$. This implies that for $x<z$, the random variable $|\omega|$ under $\mathbb{P}_{x, o, G}$ is stochastically dominated by $|\omega|$ under $\mathbb{P}_{z, o, G}$. So $L(x)=\mathbb{E}_{x, o, G}[|\omega|] \leq \mathbb{E}_{z, o, G}[|\omega|]=$ $L(z)$ for all $x<z$.

We move to prove that $P_{m}$ is a decreasing function. Write $y=(d-1) x$ and $Q_{m}(x)=\frac{d}{d-1} \mathbf{E}_{o} \sum_{k=0}^{(T \wedge m)-1} y^{k}$. Using (3.1), we have that $P_{m}(x)=Q_{m}(x) / Z(x)$. So $P_{m}^{\prime}(x)=Z(x)^{-2} \cdot\left(Q_{m}^{\prime}(x) Z(x)-Q_{m}(x) Z^{\prime}(x)\right)$.

Note that $Z=Q_{\infty}$. We have that for any $m \in \mathbb{N} \cup\{\infty\}$,

$$
y Q_{m}^{\prime}(x)=\frac{d}{d-1} \cdot \mathbf{E}_{o} \sum_{k=0}^{(T \wedge m)-1}(d-1) k y^{k}=(d-1) \mathbb{E}_{x, o, G}\left[|\omega| \mathbf{1}_{\{|\omega|<m\}}\right] \cdot Z(x) .
$$

Thus, $P_{m}^{\prime}(x) \leq 0$ if and only if

$$
\begin{aligned}
\mathbb{E}_{x, o, G}\left[|\omega| \mathbf{1}_{\{|\omega|<m\}}\right] & \leq \mathbb{E}_{x, o, G}[|\omega|] \cdot P_{m}(x) \\
& =\mathbb{E}_{x, o, G}\left[|\omega| \mathbf{1}_{\{|\omega|<m\}}\right] \cdot P_{m}(x)+\mathbb{E}_{x, o, G}\left[|\omega| \mathbf{1}_{\{|\omega| \geq m\}}\right] \cdot P_{m}(x),
\end{aligned}
$$

which is if and only if

$$
\mathbb{E}_{x, o, G}\left[|\omega| \mathbf{1}_{\{|\omega|<m\}}\right] \cdot\left(1-P_{m}(x)\right) \leq \mathbb{E}_{x, o, G}\left[|\omega| \mathbf{1}_{\{|\omega| \geq m\}}\right] \cdot P_{m}(x) .
$$

Recall that $P_{m}(x)=\mathbb{P}_{x, o, G}[|\omega|<m]$. If $P_{m}(x) \in\{0,1\}$ then the inequality above holds trivially. If $0<P_{m}(x)<1$ then the inequality is equivalent to

$$
\mathbb{E}_{x, o, G}[|\omega||| \omega \mid<m] \leq \mathbb{E}_{x, o, G}[|\omega||| \omega \mid \geq m],
$$

which is obviously true always.

Finally we prove Theorem 1.4, which states that sequences are super-critical (resp. sub-critical) if and only if $I \rightarrow 0$ (resp. $I>0$ ). 
Proof of Theorem 1.4: Jensen's inequality tells us that for any $x>0, y=(d-1) x$ and $\alpha \neq-\log y$,

$$
e^{\alpha L(x, G)} \leq \mathbb{E}_{x, G}\left[e^{\alpha|\omega|}\right]=Z_{G}(x)^{-1} \cdot \frac{d}{d-1} \cdot \mathbf{E}_{o} \sum_{k=0}^{T-1} e^{\alpha k} y^{k}=\frac{Z_{G}\left(x e^{\alpha}\right)}{Z_{G}(x)} .
$$

Now, if $\left(x_{n}\right)_{n}$ is a sub-sequence such that $Z_{G_{n}}\left(x_{n}\right) \rightarrow \infty$, then choosing $\varepsilon>0$ and $z_{n}=x_{n}(1-\varepsilon)$, we have with $\alpha=\log (1-\varepsilon)$,

$$
I\left(x_{n}, G_{n}\right)=\frac{L\left(x_{n}, G_{n}\right)+1}{Z_{G_{n}}\left(x_{n}\right)} \leq \frac{-\log (1-\varepsilon)+\log Z_{G_{n}}\left(x_{n}\right)-\log Z_{G_{n}}\left(z_{n}\right)}{-\log (1-\varepsilon) \cdot Z_{G_{n}}\left(x_{n}\right)} \rightarrow 0 .
$$

Thus, $Z_{G_{n}}\left(x_{n}\right) \rightarrow \infty$ implies that $I\left(x_{n}, G_{n}\right) \rightarrow 0$.

Also, since

$$
I\left(x_{n}, G_{n}\right)=\frac{L\left(x_{n}, G_{n}\right)+1}{Z_{G_{n}}\left(x_{n}\right)} \geq \frac{1}{Z_{G_{n}}\left(x_{n}\right)},
$$

we have that $I\left(x_{n}, G_{n}\right) \rightarrow 0$ implies that $Z_{G_{n}}\left(x_{n}\right) \rightarrow \infty$.

Thus,

$$
\begin{aligned}
\liminf _{n \rightarrow \infty} Z_{G_{n}}\left(x_{n}\right)=\infty & \Longleftrightarrow \limsup _{n \rightarrow \infty} I\left(x_{n}, G_{n}\right)=0, \\
\limsup _{n \rightarrow \infty} Z_{G_{n}}\left(x_{n}\right)<\infty & \Longleftrightarrow \liminf _{n \rightarrow \infty} I\left(x_{n}, G_{n}\right)>0 .
\end{aligned}
$$

\section{Mean-field SAW}

In this section we give a short account of the mean-field SAW; that is when $G_{n}=K_{n}$ is the complete graph on $n$ vertices.

The main (simple) observation is that on the complete graph one can count exactly the number of self-avoiding walks of a specific length. Specifically, for a fixed vertex $o_{n} \in K_{n}$,

$$
\operatorname{SAW}_{k}\left(o_{n}, K_{n}\right)= \begin{cases}\frac{(n-1) !}{(n-1-k) !} & 0 \leq k \leq n-1 \\ 0 & k>n-1 .\end{cases}
$$

Thus, for all $0 \leq k \leq n-1$,

$$
\begin{aligned}
\mathbb{P}_{x, G_{n}}[|\omega|=n-1-k] & =\frac{x^{n-1-k}\left|\operatorname{SAW}_{n-1-k}\left(o_{n}, K_{n}\right)\right|}{\sum_{j=0}^{n-1} x^{j}\left|\operatorname{SAW}_{j}\left(o_{n}, K_{n}\right)\right|} \\
& =\frac{x^{n-1-k}\left|\operatorname{SAW}_{n-1-k}\left(o_{n}, K_{n}\right)\right|}{\sum_{j=0}^{n-1} x^{n-1-j}\left|\operatorname{SAW}_{n-1-j}\left(o_{n}, K_{n}\right)\right|} \\
& =\frac{\frac{x^{-k}}{k !}}{\sum_{j=0}^{n-1} \frac{x^{-j}}{j !}}=\mathbb{P}[P=k \mid P \leq n-1],
\end{aligned}
$$

where $P \sim \operatorname{Poi}\left(x^{-1}\right)$. That is, under $\mathbb{P}_{x, K_{n}}$ the quantity $n-1-|\omega|$ has a conditional Poisson distribution. Specifically,

$$
L\left(x, K_{n}\right)=n-1-\mathbb{E}[P \mid P \leq n-1] .
$$

This leaves the task of understanding the conditional expectation above for the different regimes of $x$. 
Since this is the main observation required to prove Theorems 1.5 and 1.6, we have placed the proofs of these theorems in the appendix.

\section{SAW on large girth graphs}

We now investigate the consequences of the connections between SAW and nonbacktracking random walk made in Section 3, for sequences of graphs with large girth.

For this section let $\left(G_{n}\right)_{n}$ be a sequence of $d$-regular graphs of size $\left|G_{n}\right|=n$ and girth of $G_{n}$ being $g_{n}$. Assume that $g_{n} \rightarrow \infty$ as $n \rightarrow \infty$. We fix some vertex $o_{n} \in$ $G_{n}$, and denote $L_{n}(x)=L\left(x, o_{n}, G_{n}\right), I_{n}(x)=I\left(x, o_{n}, G_{n}\right), Z_{n}(x)=Z_{o_{n}, G_{n}}(x)$. Sometimes we will omit the subscript $n$ when it is clear from the context.

\subsection{Sub-critical phase.}

Lemma 6.1 (Sub-critical phase). Let $\left(x_{n}\right)_{n}$ be a positive sequence such that $\lim _{n \rightarrow \infty} x_{n}=x<\frac{1}{d-1}$. Then,

$$
\lim _{n \rightarrow \infty} L_{n}\left(x_{n}\right)=\frac{(d-1) x}{1-(d-1) x} .
$$

Proof: We will prove that in fact that if $x_{n}<\frac{1}{d-1}$, then for $y_{n}=(d-1) x_{n}$,

$$
\frac{y_{n}}{1-y_{n}}-\frac{g_{n} y_{n}^{g_{n}}}{1-y_{n}^{g_{n}}} \leq L_{n}\left(x_{n}\right) \leq \frac{y_{n}}{1-y_{n}} .
$$

Indeed, using (3.3), we only need to bound

$$
\frac{\mathbb{E}_{o_{n}}\left[T y^{T}\right]}{\mathbb{E}_{o_{n}}\left[1-y^{T}\right]}
$$

from above for $y<1$, where $T$ is the self intersection time of a non-backtracking random walk on $G_{n}$. Note that $T \geq g_{n}$ a.s. Also, $k \mapsto k y^{k}$ is decreasing for $k>$ $(-\log y)^{-1}$. So $\mathbb{E}_{o}\left[T y^{T}\right] \leq g_{n} y^{g_{n}}$ when $g_{n}$ is large enough, and $\mathbb{E}_{o}\left[1-y^{T}\right] \geq 1-y^{g_{n}}$.

The lemma now follows because $y_{n}<1$ for large enough $n$ and $g_{n} \rightarrow \infty$.

\subsection{Super-critical phase.}

Lemma 6.2. Suppose that $G$ is a transitive graph with girth $g$ and size $|G|=n$. Let

$$
\tau=\min \left\{t: \max _{u, v}\left|\mathbf{P}_{u}[\mathrm{NB}(t)=v]-\frac{1}{n}\right| \leq \frac{1}{2 n}\right\}
$$

be the mixing time of the non-backtracking random walk NB on $G$, and let $T$ be the self intersection time of NB. For all $m \geq \tau$,

$$
\mathbf{P}_{o}[T>k+m] \geq\left(1-\frac{3(k+1) m}{2 n}-m^{2} \cdot(d-1)^{-\left\lfloor g_{n} / 2\right\rfloor}\right) \cdot \mathbf{P}_{o}[T>k] .
$$

Proof: We will make use of the fact that uniformly over $v$,

$$
\mathbf{P}_{v}[\mathrm{NB}(t)=v] \leq \begin{cases}0 & \text { if } t<g_{n} \\ (d-1)^{-\left\lfloor g_{n} / 2\right\rfloor} & \text { if } g_{n} \leq t<\tau \\ \frac{3}{2 n} & \text { if } t \geq \tau\end{cases}
$$


The middle case coming from the fact that $\left\{u: \operatorname{dist}(v, u) \leq \frac{g_{n}}{2}\right\}$ is a tree, so to hit $v$ at time $t$ with a non-backtracking walk, one must be at distance $\left\lfloor\frac{g_{n}}{2}\right\rfloor$ at time $t-\left\lfloor\frac{g_{n}}{2}\right\rfloor$ and then move towards $v$ in the next $\left\lfloor\frac{g_{n}}{2}\right\rfloor$ steps.

Fix $m \geq \tau$. NB $[0, k+m]$ is uniformly distributed on non-backtracking paths of length $k+m$ starting at $\mathrm{NB}(0)$. Thus, conditioned on $\mathrm{NB}[0, k], \mathrm{NB}(k+m)=v$, the walk $\mathrm{NB}[k, k+m]$ is uniformly distributed on non-backtracking paths of length $m$ starting at $\mathrm{NB}(k)$, such that the first step is not $\mathrm{NB}(k-1)$ and such that the last step is at $v$. Fix $v$ and conditioned on $\mathrm{NB}[0, k]=A$. Set

$$
\begin{gathered}
\Omega=\Omega(v, A)=\left\{\omega \text { non-backtracking: } \begin{array}{l}
|\omega|=m, \omega(0)=\mathrm{NB}(k), \omega(1) \neq \mathrm{NB}(k-1), \\
\omega[1, m] \cap A \neq \emptyset, \omega(m)=v
\end{array}\right\} \\
\Omega^{\prime}=\Omega^{\prime}(v, A)=\left\{\begin{array}{ll}
\omega \text { non-backtracking }: \begin{array}{l}
|\omega|=m, \omega(0)=v, \\
\omega[0, m-1] \cap A \neq \emptyset
\end{array}
\end{array}\right\}
\end{gathered}
$$

Since for every $\omega \in \Omega$ we have that the reversal of $\omega$ is in $\Omega^{\prime}$ we get that $|\Omega| \leq\left|\Omega^{\prime}\right|$. Thus, using the fact that $m \geq \tau$,

$$
\begin{aligned}
\mathbf{P}_{o}[\mathrm{NB}[k+1, k+m] & \cap A \neq \emptyset, \mathrm{NB}[0, k]=A, \mathrm{NB}(k+m)=v] \\
& =|\Omega| \cdot(d-1)^{-m} \cdot \mathbf{P}_{o}[\mathrm{NB}[0, k]=A, \mathrm{NB}(k+m)=v] \\
& \leq\left|\Omega^{\prime}\right| \cdot(d-1)^{-m} \cdot \mathbf{P}_{o}[\mathrm{NB}[0, k]=A, \mathrm{NB}(k+m)=v] \\
& =\mathbf{P}_{v}[\mathrm{NB}[0, m-1] \cap A \neq \emptyset] \cdot \mathbf{P}_{o}[\mathrm{NB}[0, k]=A, \mathrm{NB}(k+m)=v] \\
& \leq \mathbf{P}_{v}[\mathrm{NB}[0, m-1] \cap A \neq \emptyset] \cdot \mathbf{P}_{o}[\mathrm{NB}[0, k]=A] \cdot \frac{3}{2 n} .
\end{aligned}
$$

Summing over $v$ we obtain

$$
\mathbf{P}_{o}[\mathrm{NB}[k+1, k+m] \cap A \neq \emptyset \mid \mathrm{NB}[0, k]=A] \leq \frac{3}{2} \cdot \mathbf{P}_{o}[\mathrm{NB}[0, m-1] \cap A \neq \emptyset] .
$$

Since the uniform distribution is stationary for the non-backtracking random walk on a regular graph,

$$
\begin{aligned}
\frac{1}{n} \sum_{o \in G} \mathbf{P}_{o}[\mathrm{NB}[0, m-1] \cap A \neq \emptyset] & \leq \frac{1}{n} \sum_{o \in G} \mathbf{E}_{o}[|\mathrm{NB}[0, m-1] \cap A|] \\
& =\frac{1}{n} \sum_{t=0}^{m-1} \sum_{o \in G} \mathbf{P}_{o}[\mathrm{NB}(t) \in A]=\frac{|A| m}{n} .
\end{aligned}
$$

Thus,

$$
\frac{1}{n} \sum_{o \in G} \mathbf{P}_{o}[\mathrm{NB}[k+1, k+m] \cap \mathrm{NB}[0, k] \neq \emptyset \mid \mathrm{NB}[0, k]] \leq \frac{3(k+1) m}{2 n} .
$$

Also, if $\mathrm{NB}[k, k+m] \notin \mathrm{SAW}$ then there exist $0 \leq t<t^{\prime} \leq m$ such that $\mathrm{NB}(k+t)=$ $\mathrm{NB}\left(k+t^{\prime}\right)$. We have seen above that for any such pair $t<t^{\prime}$, this probability is bounded by $\mathbf{P}_{o}\left[\mathrm{NB}(k+t)=\mathrm{NB}\left(k+t^{\prime}\right) \mid \mathrm{NB}[0, k]\right] \leq(d-1)^{-\left\lfloor g_{n} / 2\right\rfloor}$. Thus,

$$
\begin{aligned}
\mathbf{P}_{o}[\mathrm{NB}[k, k+m] \notin \mathrm{SAW} \mid \mathrm{NB}[0, k]] & \leq \sum_{\substack{0 \leq t<t^{\prime} \leq m\\
}} \mathbb{P}\left[\mathrm{NB}(k+t)=\mathrm{NB}\left(k+t^{\prime}\right) \mid \mathrm{NB}[0, k]\right] \\
& \leq m^{2} \cdot(d-1)^{-\left\lfloor g_{n} / 2\right\rfloor} .
\end{aligned}
$$


If $\mathrm{NB}[k, k+m] \in \mathrm{SAW}, \mathrm{NB}[0, k] \in \mathrm{SAW}$ and $\mathrm{NB}[k+1, k+m] \cap \mathrm{NB}[0, k]=\emptyset$, then $\mathrm{NB}[0, k+m] \in \mathrm{SAW}$. Thus, combining all the above we obtain that

$$
\begin{aligned}
& \frac{1}{n} \sum_{o \in G} \mathbf{P}_{o}[\mathrm{NB}[0, k+m] \in \mathrm{SAW}] \\
& \geq\left(1-\frac{3(k+1) m}{2 n}-m^{2} \cdot(d-1)^{-\left\lfloor g_{n} / 2\right\rfloor}\right) \cdot \frac{1}{n} \sum_{o \in G} \mathbf{P}_{o}[\mathrm{NB}[0, k] \in \mathrm{SAW}] .
\end{aligned}
$$

Since $G$ is transitive, $\mathbb{P}_{o}[\mathrm{NB}[0, k] \in \mathrm{SAW}]$ does not depend on the choice of starting vertex $o \in G$, and we obtain the lemma.

Corollary 6.3. Let $\left(G_{n}\right)_{n}$ be a sequence of transitive graphs such that $\left|G_{n}\right| \nearrow \infty$. Suppose that the mixing time of the non-backtracking random walk on $G_{n}$ satisfies $\tau_{n}=o\left((d-1)^{g_{n} / 4}\right)$ as $n \rightarrow \infty$, where $g_{n}$ is the girth of $G_{n}$.

Then, there exists a constant $c>0$ such that for every $\delta>0$ and all $k \leq \frac{\delta}{6}\left|G_{n}\right|$, $\mathbf{P}_{o}[T>k] \geq c e^{-\delta k}$.

Proof: Set $p_{k}=\mathbf{P}_{o}[T>k]$. If we choose $m=m_{n}=\tau_{n}$, we may take $n$ large enough so that $m^{2} \cdot(d-1)^{-\left\lfloor g_{n} / 2\right\rfloor}<\frac{1}{4}$. By Lemma 6.2 (using the inequality $e^{-2 \xi} \leq 1-\xi$ valid for all $\left.\xi \leq \frac{1}{4}\right)$,

$$
\begin{aligned}
p_{k} & \geq \exp \left(-2 m^{2} \cdot(d-1)^{-\left\lfloor g_{n} / 2\right\rfloor}-\frac{3(k-m) m}{\left|G_{n}\right|}-\frac{3 m}{\left|G_{n}\right|}\right) \cdot p_{k-m} \\
& \geq \cdots \geq \exp \left(-\left\lfloor\frac{k}{m}\right\rfloor \cdot 2 m^{2} \cdot(d-1)^{-\left\lfloor g_{n} / 2\right\rfloor}-\frac{3 m}{\left|G_{n}\right|} \cdot \sum_{j=1}^{\lfloor k / m\rfloor} j m-\frac{3 m}{\left|G_{n}\right|} \cdot\left\lfloor\frac{k}{m}\right\rfloor\right) \cdot p_{m} \\
& \geq \exp \left(-2 k m \cdot(d-1)^{-\left\lfloor g_{n} / 2\right\rfloor}-\frac{3 k^{2}}{\left|G_{n}\right|}-\frac{3 k}{\left|G_{n}\right|}\right) \cdot \exp \left(-2 m^{2} \cdot(d-1)^{-\left\lfloor g_{n} / 2\right\rfloor}\right) .
\end{aligned}
$$

Thus, if $k \leq \beta\left|G_{n}\right|$ then,

$$
p_{k} \geq e^{-1 / 2} \cdot \exp \left(-\frac{1}{2 m} k-3 \beta k-\frac{3}{\left|G_{n}\right|} k\right) .
$$

Since $m=\tau_{n} \rightarrow \infty$ as $n \rightarrow \infty$, we have that if $\beta=\frac{\delta}{6}$ then for all $n$ large enough (so that $2 m>>\delta^{-1}$ ), $p_{k} \geq e^{-1 / 2} \cdot e^{-\delta k}$.

Lemma 6.4 (Super-critical phase). Let $\left(G_{n}\right)_{n}$ be a sequence of transitive graphs such that $\left|G_{n}\right| \nearrow \infty$ with girth $g_{n}$. Suppose that the mixing time of the nonbacktracking random walk on $G_{n}$ satisfies $\tau_{n}=o\left((d-1)^{g_{n} / 4}\right)$ as $n \rightarrow \infty$, where $g_{n}$ is the girth of $G_{n}$.

Then, there exists a constant $c>0$ such that for any $x>\frac{1}{d-1}$ there exists $n_{0}>0$ such that for all $n>n_{0}$,

$$
L_{n}(x) \geq c(1 \wedge \log ((d-1) x)) \cdot\left|G_{n}\right| .
$$

Proof: Set $y=(d-1) x>1$. Choose $\delta>0$ small enough so that $\delta=1 \wedge \frac{1}{2} \log y$. For any integers $0<m<M \leq \frac{\delta}{6}\left|G_{n}\right|$ we have by Corollary 6.3,

$$
\begin{aligned}
& \sum_{k=0}^{m} y^{k} \mathbf{P}_{o}[T>k] \leq \frac{y^{m+1}-1}{y-1}, \\
& \sum_{k=0}^{M} y^{k} \mathbf{P}_{o}[T>k] \geq c \cdot \sum_{k=0}^{M} \sqrt{y}^{k}=c \cdot \frac{\sqrt{y}^{M+1}-1}{\sqrt{y}-1} .
\end{aligned}
$$


Choose $\left.M=\left\lfloor\frac{\delta}{6} \mid G_{n}\right\rfloor\right\rfloor$ and $m=\left\lfloor\frac{M-1}{4}\right\rfloor$, so that $y^{m+1} \sqrt{y}^{-M-1} \leq y^{-m}$. Note that for some universal constant $c>0$ we have that $m \geq c \delta\left|G_{n}\right|$. We can compute:

$$
\begin{aligned}
\mathbb{P}_{x, o_{n}, G_{n}}[|\omega| \leq m] & =\frac{\sum_{k=0}^{m} y^{k} \mathbf{P}_{o}[T>k]}{\sum_{k=0}^{\left|G_{n}\right|-1} y^{k} \mathbf{P}_{o}[T>k]} \leq c^{-1} \cdot \frac{y^{m+1}-1}{\sqrt{y}^{M+1}-1} \cdot \frac{\sqrt{y}-1}{y-1} \\
& \leq c^{-1} \cdot \frac{y^{m+1}}{\sqrt{y}^{M+1}} \cdot \frac{1}{1-\sqrt{y}^{-M-1}} \leq C \cdot y^{-c \delta\left|G_{n}\right|},
\end{aligned}
$$

for some constant $C>0$. Thus,

$$
L_{n}(x) \geq\left(1-C y^{-c \delta\left|G_{n}\right|}\right) \cdot c \delta\left|G_{n}\right| .
$$

\subsection{Critical phase.}

Lemma 6.5 (Critical phase). For a sequence of d-regular transitive graphs $\left(G_{n}\right)_{n}$, of size $\left|G_{n}\right|=n$ and girth $g_{n} \rightarrow \infty$ as $n \rightarrow \infty$, we have that at the critical sequence $x_{n}=\frac{1}{d-1}$,

$$
\frac{1}{2} \mathbf{E}_{o}[T-1] \leq L_{n}\left(x_{n}\right) \leq \frac{d}{d-1} \mathbf{E}_{o}[T]-1
$$

Specifically, $L_{n}\left(x_{n}\right) \rightarrow \infty$ and $L_{n}\left(x_{n}\right) \leq \frac{d}{d-1}(1+o(1)) g_{n}(d-1)^{g_{n}}$. So $L_{n}$ has neither sub-critical nor super-critical behavior.

Proof: Using (3.4) and (3.5) we have that $Z_{n}\left(\frac{1}{d-1}\right)=\frac{d}{d-1} \mathbf{E}_{o}[T] \rightarrow \infty$, and

$$
L_{n}\left(\frac{1}{d-1}\right)=\frac{\mathbf{E}_{o}\left[T^{2}\right]-\mathbf{E}_{o}[T]}{2 \mathbf{E}_{o}[T]} \geq \frac{1}{2} \mathbf{E}_{o}[T]-\frac{1}{2} \rightarrow \infty,
$$

where $T$ is the self intersection time of a non-backtracking random walk on $G_{n}$

Proposition 1.9 tells us that $L_{n}(x)+1 \leq Z_{n}(x)$, so that $L_{n}\left(\frac{1}{d-1}\right) \leq \frac{d}{d-1} \mathbf{E}_{o}[T]-1$.

Since $g_{n}$ is the girth of $G_{n}$, we have $\mathbf{P}_{v}\left[\operatorname{NB}\left(g_{n}\right)=v\right] \geq(d-1)^{-g_{n}}$. Thus, for any $j \leq k-g_{n}$,

$$
\mathbf{P}\left[\mathrm{NB}\left(j+g_{n}\right)=\mathrm{NB}(j) \mid \mathrm{NB}[0, j]\right] \geq(d-1)^{-g_{n}} .
$$

Thus, for $k>g_{n}$,

$$
\begin{aligned}
\mathbf{P}_{o}[T>k] & \leq \mathbf{P}_{o}\left[T>k-g_{n}\right] \cdot \mathbf{P}\left[\mathrm{NB}(k) \neq \mathrm{NB}\left(k-g_{n}\right) \mid \mathrm{NB}\left[0, k-g_{n}\right]\right] \\
& \leq \mathbf{P}_{o}\left[T>k-g_{n}\right] \cdot\left(1-(d-1)^{-g_{n}}\right) \\
& \leq \cdots \leq\left(1-(d-1)^{-g_{n}}\right)^{\left\lfloor k / g_{n}\right\rfloor} .
\end{aligned}
$$

This easily shows that

$$
\mathbf{E}_{o}[T] \leq \frac{1}{\left(1-(d-1)^{-g_{n}}\right) \cdot\left(1-\left(1-(d-1)^{-g_{n}}\right)^{1 / g_{n}}\right)} \leq(1+o(1)) \cdot g_{n}(d-1)^{g_{n}},
$$

(using the inequalities $1-\xi \leq e^{-\xi}$ and $1-\frac{1}{2} \xi \geq e^{-\xi}$, valid $0<\xi<\frac{1}{2}$ ).

Remark 6.6. By the proof of Corollary 6.3, as long as $\tau_{n}=o\left((d-1)^{g_{n} / 4}\right)$ as $n \rightarrow \infty$, taking $\beta=\frac{k}{\left|G_{n}\right|}$ in (6.2), we have that there exists a constant $c>0$ such that

$$
\mathbf{P}_{o}[T>k] \geq c \exp \left(-k \cdot\left(\frac{1}{2 \tau_{n}}+\frac{3(k+1)}{\left|G_{n}\right|}\right)\right)
$$

where $\tau_{n}$ is the mixing time of the non-backtracking random walk. If we take $k=$ $\left\lfloor\min \left\{\tau_{n}, \sqrt{\left|G_{n}\right|}\right\}\right\rfloor$ we get that for some universal constant $c^{\prime}>0, \mathbf{P}_{o}[T>k] \geq c^{\prime}$, which implies that $\mathbf{E}_{o}[T] \geq c^{\prime} \min \left\{\tau_{n}, \sqrt{\left|G_{n}\right|}\right\}$ in this case. 
6.4. Proofs of theorems for large girth graphs.

Proof of Theorem 1.7: This is just a combination of Lemmas 6.1, 6.4 and 6.5.

Finally we prove Theorem 1.8, which computes the critical exponent $\gamma$ for large girth graphs.

Proof of Theorem 1.8: Let $T$ be the self intersection time of a non-backtracking random walk on $G_{n}$. Note that since $T \geq g_{n}$ a.s., which converges to infinity, we get using (3.4) and (3.5) $Z_{G_{n}}\left(\frac{1}{d-1}\right)=\frac{d}{d-1} \mathbf{E}_{o}[T] \rightarrow \infty$, and

$$
L\left(\frac{1}{d-1}, G_{n}\right)=\frac{\mathbf{E}_{o}\left[T^{2}\right]-\mathbf{E}_{o}[T]}{2 \mathbf{E}_{o}[T]} \geq \frac{1}{2} \mathbf{E}_{o}[T]-\frac{1}{2} \rightarrow \infty .
$$

So for the critical sequence $x_{n}=\frac{1}{d-1}$,

$\lim _{n \rightarrow \infty} \frac{\log Z_{G_{n}}\left(x_{n}\right)}{\log \left(L\left(x_{n}, G_{n}\right)+1\right)} \leq \lim _{n \rightarrow \infty} \frac{\log \frac{d}{d-1}+\log \mathbf{E}_{o}[T]}{\log \mathbf{E}_{o}[T]+\log \left(\mathbf{E}_{o}[T]+1\right)-\log \mathbf{E}_{o}[T]-\log 2}=1$.

The inequality $\gamma \geq 1$ is immediate from Proposition 1.9, since $L\left(x_{n}, G_{n}\right)+1 \leq$ $Z_{G_{n}}\left(x_{n}\right)$.

The sub-critical case $x_{n} \rightarrow x<\frac{1}{d-1}$ just follows from plugging in the values of $L, Z$ from (3.2) and (3.3).

For the super-critical case $x_{n} \rightarrow x>\frac{1}{d-1}$, as long as $\tau_{n}=o\left((d-1)^{g_{n} / 4}\right)$ we can duplicate the super-critical case from the proof of Theorem 1.6: Set $z_{n}=$ $\frac{1}{2} \cdot\left(\frac{1}{d-1}+x_{n}\right)$, so that $z_{n} \rightarrow z:=\frac{1}{2}\left(\frac{1}{d-1}+x\right)>\frac{1}{d-1}$. By Lemma 6.4 , for any $w$ we have

$$
L\left(z_{n}, G_{n}\right) \geq c\left(1 \wedge \log \left((d-1) z_{n}\right)\right) \cdot\left|G_{n}\right| \geq c\left(1 \wedge \log \left((d-1) z_{n}\right)\right) \cdot L\left(w, G_{n}\right) .
$$

Thus,

$$
\lim _{n \rightarrow \infty} \frac{L\left(z_{n}, G_{n}\right)}{\log \left(L\left(z_{n}, G_{n}\right)+1\right)}=\infty \quad \text { and } \quad \lim _{n \rightarrow \infty} \frac{\log L\left(x_{n}, G_{n}\right)}{\log L\left(z_{n}, G_{n}\right)}=1 .
$$

Consider $f(x):=\log Z_{G_{n}}(x)$. Note that $x f^{\prime}(x)=L\left(x, G_{n}\right)$. So we have that for $x>z$, there exists $w \in[z, x]$ such that

$$
\log Z_{G_{n}}(x)-\log Z_{G_{n}}(z)=f^{\prime}(w)(x-z)=\frac{1}{w} L\left(w, G_{n}\right)(x-z) \geq \frac{x-z}{x} \cdot L\left(z, G_{n}\right) .
$$

Thus, for $x_{n}, z_{n}$ as above,

$$
\lim _{n \rightarrow \infty} \frac{\log Z_{G_{n}}\left(x_{n}\right)}{\log \left(L\left(x_{n}, G_{n}\right)+1\right)} \geq 1+\lim _{n \rightarrow \infty} \frac{\left(x_{n}-z_{n}\right) \cdot L\left(z_{n}, G_{n}\right)}{x_{n} \log \left(L\left(z_{n}, G_{n}\right)+1\right)}=\infty .
$$

\section{Appendix A. Complete graph proofs}

A.1. Mean field super-critical regime. First a classical large deviations argument, which we include for completeness.

Proposition A.1. Let $P \sim \operatorname{Poi}\left(x^{-1}\right)$. Then, for all $n>x^{-1}$,

$$
\mathbb{P}[P \geq n] \leq(x n)^{-n} \cdot e^{n-\frac{1}{x}} .
$$


Proof: For any $\lambda>0$, the Laplace transform of $P$ is

$$
\mathbb{E}\left[e^{\lambda P}\right]=\exp \left(x^{-1} \cdot\left(e^{\lambda}-1\right)\right) .
$$

Thus, for any $\lambda>0$,

$$
\mathbb{P}[P \geq n] \leq \mathbb{E}\left[e^{\lambda P}\right] e^{-\lambda n}=\exp \left(x^{-1} \cdot\left(e^{\lambda}-1\right)-\lambda n\right) .
$$

Minimizing the right hand side above over $\lambda$, we obtain $\lambda=\log (x n)$ (which is good since we assumed $x n>1$ ), so

$$
\mathbb{P}[P \geq n] \leq\left(\frac{e}{x n}\right)^{n} \cdot e^{-1 / x} .
$$

Lemma A.2. For $\varepsilon>0$ and $x=\frac{1+\varepsilon}{n-1}$, we have

$$
\left|L\left(x, K_{n}\right)-\frac{\varepsilon}{1+\varepsilon}(n-1)\right| \leq \frac{n I(\varepsilon)^{n / 2}}{1-I(\varepsilon)^{n}},
$$

where $I(\varepsilon)=\max \left\{e^{-\varepsilon^{2} / 8}, \sqrt{e} / 2\right\}$. Specifically, if $\left(\varepsilon_{n}\right)_{n}$ is a sequence converging to $\mathcal{E} \in[0, \infty]$ such that $n \cdot \varepsilon_{n}^{2} \rightarrow \infty$ as $n \rightarrow \infty$, then for the sequence $x_{n}=\frac{1+\varepsilon_{n}}{n}$,

$$
\lim _{n \rightarrow \infty} \frac{L\left(x_{n}, K_{n}\right)}{n-1}=\frac{\mathcal{E}}{1+\mathcal{E}},
$$

where $\frac{\infty}{1+\infty}=1$.

Proof: Let $P \sim \operatorname{Poi}\left(x^{-1}\right)$, and let $Q \sim \operatorname{Poi}\left(\frac{n}{1+\varepsilon}\right)$. So $P$ is stochastically dominated by $Q$. Thus, by Proposition A.1,

$$
\mathbb{P}[P \geq n] \leq \mathbb{P}[Q \geq n] \leq(1+\varepsilon)^{-n} e^{n} e^{-\frac{n}{1+\varepsilon}}=\exp \left(\frac{\varepsilon}{1+\varepsilon} n\right) \cdot(1+\varepsilon)^{-n} .
$$

We use the inequality $e^{\xi} \leq 1+\xi+\frac{\xi^{2}}{2} e^{\xi}$, so if $\xi<1$, then $e^{\xi} \leq \frac{1+\xi}{1-\xi^{2} / 2}$. Plugging in $\xi=\frac{\varepsilon}{1+\varepsilon}$, we obtain that when $\varepsilon<1$,

$$
\exp \left(\frac{\varepsilon}{1+\varepsilon}\right)(1+\varepsilon)^{-1} \leq \frac{1+2 \varepsilon}{1+2 \varepsilon+\varepsilon^{2} / 2} \leq 1-\frac{\varepsilon^{2}}{8}
$$

and if $\varepsilon \geq 1$ then since $\xi e^{-\xi}$ increases when $0<\xi<1$, with $\xi=\frac{1}{1+\varepsilon}$ we get

$$
\exp \left(\frac{\varepsilon}{1+\varepsilon}\right)(1+\varepsilon)^{-1} \leq e \cdot \xi e^{-\xi} \leq \frac{\sqrt{e}}{2} .
$$

So we get that $\mathbb{P}[P \geq n] \leq I(\varepsilon)^{n}$. Thus, for some $c=c(\varepsilon)$,

$$
\mathbb{E}[P \mid P \leq n-1] \leq \frac{\mathbb{E}[P]}{1-\mathbb{P}[P \geq n]} \leq \frac{x^{-1}}{1-I(\varepsilon)^{n}} \leq \frac{n-1}{1+\varepsilon} \cdot\left(1+\frac{I(\varepsilon)^{n}}{1-I(\varepsilon)^{n}}\right) .
$$

We use this bound with (5.1) to obtain that for $x \geq \frac{1+\varepsilon}{n-1}$,

$$
\begin{aligned}
L\left(x, K_{n}\right) & =n-1-\mathbb{E}[P \mid P \leq n-1] \geq(n-1) \cdot\left(1-\frac{1}{1+\varepsilon} \cdot\left(1+\frac{I(\varepsilon)^{n}}{1-I(\varepsilon)^{n}}\right)\right) \\
& \geq \frac{\varepsilon(n-1)}{1+\varepsilon}-\frac{n I(\varepsilon)^{n}}{1-I(\varepsilon)^{n}} .
\end{aligned}
$$

This proves the lower bound on $L\left(x, K_{n}\right)$. 
For the upper bound, note that by Cauchy-Schwarz

$$
\begin{aligned}
\mathbb{E}\left[P 1_{\{P \geq n\}}\right] & \leq \sqrt{\mathbb{E}\left[P^{2}\right] \cdot \mathbb{P}[P \geq n]} \leq \sqrt{x^{-2}+x^{-1}} \cdot I(\varepsilon)^{n / 2} \\
& \leq n \cdot I(\varepsilon)^{n / 2} .
\end{aligned}
$$

Thus,

$$
\begin{aligned}
L\left(x, K_{n}\right) & =n-1-\mathbb{E}[P \mid P \leq n-1]=n-1-\frac{\mathbb{E}[P]-\mathbb{E}\left[P \mathbf{1}_{\{P \geq n\}}\right]}{1-\mathbb{P}[P \geq n]} \\
& \leq n-1-\mathbb{E}[P]+\frac{\mathbb{E}\left[P \mathbf{1}_{\{P \geq n\}}\right]}{1-\mathbb{P}[P \geq n]} \leq \frac{\varepsilon(n-1)}{1+\varepsilon}+\frac{n I(\varepsilon)^{n / 2}}{1-I(\varepsilon)^{n}}
\end{aligned}
$$

(In the second inequality is where we use that $\mathbb{E}[P]=x^{-1}=\frac{n-1}{1+\varepsilon}$.)

Remark A.3. Note that Lemma A.2 gives more than required for the super-critical phase in Theorem 1.5. For $\varepsilon_{n}>n^{-1 / 2}$, we have that the expected length $L\left(x, K_{n}\right)$ is very near $\frac{\varepsilon_{n}(n-1)}{1+\varepsilon_{n}}$ as $n \rightarrow \infty$.

\section{A.2. Mean field critical regime.}

Lemma A.4. There exists a constant $\alpha>0$ such that for $x=x_{n}=\frac{1}{n-1}$,

$$
\lim _{n \rightarrow \infty} \frac{L\left(x_{n}, K_{n}\right)}{\sqrt{n-1}}=\alpha .
$$

Proof: Let $\left(P_{k}\right)_{k}$ be i.i.d. Poisson-1 random variables, and let $P=\sum_{k=1}^{n-1} P_{k}$. So $P \sim \operatorname{Poi}\left(x^{-1}\right)$. Since $\mathbb{E}[P]=n-1$ and $\operatorname{Var}[P]=n-1$ we have by the central limit theorem that the sequence $X_{n}:=\frac{P-(n-1)}{\sqrt{n-1}}$ converges in distribution to a standard Gaussian $N \sim \mathcal{N}(0,1)$

From this, a simple application of the Portmanteau Theorem gives that

$$
\frac{\mathbb{E}[P \mid P \leq n-1]-(n-1)}{\sqrt{n-1}}=\mathbb{E}\left[X_{n} \mid X_{n} \leq 0\right] \rightarrow \mathbb{E}[N \mid N \leq 0] .
$$

So setting $\alpha:=-\mathbb{E}[N \mid N \leq 0]=\mathbb{E}|N|>0$ we have that when $x=\frac{1}{n-1}$,

$$
\frac{1}{\sqrt{n-1}} \cdot L\left(x_{n}, K_{n}\right) \rightarrow \alpha
$$

\section{A.3. Mean field sub-critical regime.}

Lemma A.5. For any $\varepsilon, \delta>0$ there exists $n_{0}>0$ such that for all $n>n_{0}$, if $x=\frac{1-\varepsilon}{n-1}$ then,

$$
\frac{1-\varepsilon}{\varepsilon} \cdot e^{-\delta} \cdot\left(1-\frac{3}{n}\right) \leq L\left(x, K_{n}\right) \leq \frac{1-\varepsilon}{\varepsilon} .
$$

Consequently, if $\left(x_{n}\right)_{n}$ is such that $\lim _{n \rightarrow \infty}\left(n x_{n}\right)=1-\varepsilon$ then

$$
\lim _{n \rightarrow \infty} L\left(x_{n}, K_{n}\right)=\frac{1-\varepsilon}{\varepsilon} .
$$

Proof: For the upper bound we use (3.3) to deduce that

$$
L\left(x, K_{n}\right) \leq \frac{x(n-2)}{1-x(n-2)} \leq \frac{\varepsilon}{1-\varepsilon} .
$$


For the lower bound, by (3.2),

$$
Z_{K_{n}}(x) \leq \frac{n-1}{n-2} \cdot \frac{1}{1-x(n-2)} \leq \frac{n-1}{n-2} \cdot \frac{1}{\varepsilon} .
$$

Note that $1-\xi \geq e^{-2 \xi}$ for $\xi \leq \frac{1}{4}$, so for any fixed $\delta>0$,

$$
\begin{aligned}
L\left(x, K_{n}\right) & =Z_{K_{n}}(x)^{-1} \cdot \sum_{k=0}^{n-1} k x^{k}\left|\operatorname{SAW}_{k}\left(K_{n}\right)\right| \geq \varepsilon \cdot \frac{n-2}{n-1} \cdot \sum_{k \leq(n-1) / 4} k(1-\varepsilon)^{k} \prod_{j=0}^{k-1}\left(1-\frac{j}{n-1}\right) \\
& \geq \varepsilon \cdot \frac{n-2}{n-1} \cdot \sum_{k \leq(n-1) / 4} k(1-\varepsilon)^{k} \exp \left(-\frac{k^{2}}{n-1}\right) \geq \frac{n-2}{n-1} \cdot e^{-\delta} \cdot \sum_{k \leq \sqrt{\delta(n-1)}} k(1-\varepsilon)^{k} \varepsilon \\
& \geq e^{-\delta} \cdot \frac{n-2}{n-1} \cdot \frac{1-\varepsilon}{\varepsilon} \cdot\left(1-(1-\varepsilon)^{\sqrt{\delta(n-1)}}\right) .
\end{aligned}
$$

Taking $n \rightarrow \infty$ we have that for all $\delta>0$,

$$
\lim _{n \rightarrow \infty} L\left(\frac{1-\varepsilon}{n-1}, K_{n}\right) \geq e^{-\delta} \cdot \frac{1-\varepsilon}{\varepsilon},
$$

so if $\left(x_{n}\right)_{n}$ is such that $\lim _{n \rightarrow \infty}\left(n x_{n}\right)=1-\varepsilon$ then

$$
\lim _{n \rightarrow \infty} L\left(x_{n}, K_{n}\right)=\frac{1-\varepsilon}{\varepsilon} .
$$

\section{A.4. Proofs of theorems for mean field case.}

Proof of Theorem 1.5: Combining of Lemmas A.2, A.4 and A.5 we have the asymptotics for the expected length $L$.

By Proposition 1.9, we get that for any $\varepsilon>0$,

$$
Z_{K_{n}}\left(\frac{1+\varepsilon}{n}\right) \geq L\left(\frac{1+\varepsilon}{n}, K_{n}\right)+1 \rightarrow \infty,
$$

by Lemma A.2, and

$$
Z_{K_{n}}\left(\frac{1-\varepsilon}{n}\right) \leq \frac{1}{1-(1-\varepsilon) \frac{n-2}{n}} \rightarrow \frac{1}{\varepsilon}<\infty
$$

by $(3.2)$.

This implies that $\left(\frac{1}{n}\right)_{n}$ is a critical sequence, and that in the super-critical case $I\left(x_{n}, G_{n}\right) \rightarrow 0$.

Also, in the sub-critical case where $n x_{n} \rightarrow 1-\varepsilon$, we have that

$$
I\left(x_{n}, K_{n}\right)=\frac{L\left(x_{n}, K_{n}\right)+1}{Z_{K_{n}}\left(x_{n}\right)} \geq\left(L\left(x_{n}, K_{n}\right)+1\right) \cdot\left(1-(1-\varepsilon) \frac{n-2}{n}\right) \rightarrow 1 .
$$

We now prove Theorem 1.6, calculating $\gamma$ for sequences in the case where $G_{n}=$ $K_{n}$, the complete graph on $n$ vertices.

Proof of Theorem 1.6: We start with the critical case $x_{n}=\frac{1}{n-2}$. In this case we have by (3.4) and (3.5) that

$$
\frac{n-2}{2(n-1)} \cdot Z_{G_{n}}\left(x_{n}\right)=\frac{1}{2} \mathbf{E}_{o}[T] \leq \frac{\mathbf{E}_{o}\left[T^{2}+T\right]}{2 \mathbf{E}_{o}[T]}=L\left(x_{n}, G_{n}\right)+1 \leq Z_{G_{n}}\left(x_{n}\right) .
$$


Since $\mathbf{E}_{o}[T] \rightarrow \infty$

$$
\lim _{n \rightarrow \infty} \frac{\log Z_{G_{n}}\left(x_{n}\right)}{\log \left(L\left(x_{n}, G_{n}\right)+1\right)}=1 .
$$

In the sub-critical case where $\left(n x_{n}\right) \rightarrow 1-\varepsilon$, we have by (3.2)

$$
Z_{G_{n}}\left(x_{n}\right) \leq \frac{n-1}{(n-2)\left(1-(n-2) x_{n}\right)} \rightarrow \frac{1}{\varepsilon},
$$

and $L\left(x_{n}, G_{n}\right)+1 \rightarrow \frac{1}{\varepsilon}$, so in this case as well,

$$
\lim _{n \rightarrow \infty} \frac{\log Z_{G_{n}}\left(x_{n}\right)}{\log \left(L\left(x_{n}, G_{n}\right)+1\right)}=1 .
$$

Finally, in the super-critical case $\left(n x_{n}\right) \rightarrow 1+\varepsilon$, set $z_{n}=\frac{1}{2} \cdot\left(\frac{1}{n}+x_{n}\right)$. Then $n z_{n} \rightarrow z:=1+\frac{1}{2} \varepsilon$, and we have by Lemma A.2 that as $n \rightarrow \infty$,

$$
L\left(z_{n}, G_{n}\right) \geq(1-o(1)) \frac{\varepsilon}{2+\varepsilon} \cdot n \geq(1-o(1)) \frac{\varepsilon}{2+\varepsilon} \cdot L\left(x_{n}, G_{n}\right) .
$$

Thus,

$$
\lim _{n \rightarrow \infty} \frac{L\left(z_{n}, G_{n}\right)}{\log \left(L\left(z_{n}, G_{n}\right)+1\right)}=\infty \quad \text { and } \quad \lim _{n \rightarrow \infty} \frac{\log L\left(x_{n}, G_{n}\right)}{\log L\left(z_{n}, G_{n}\right)}=1 .
$$

Consider $f(x):=\log Z_{G_{n}}(x)$. Note that $x f^{\prime}(x)=L\left(x, G_{n}\right)$. So we have that for $x>z$, there exists $w \in[z, x]$ such that

$$
\log Z_{G_{n}}(x)-\log Z_{G_{n}}(z)=f^{\prime}(w)(x-z)=\frac{1}{w} L\left(w, G_{n}\right)(x-z) \geq \frac{x-z}{x} \cdot L\left(z, G_{n}\right) .
$$

Thus, for $x_{n}, z_{n}$ as above,

$$
\begin{aligned}
\lim _{n \rightarrow \infty} & \frac{\log Z_{G_{n}}\left(x_{n}\right)}{\log \left(L\left(x_{n}, G_{n}\right)+1\right)} \\
& \geq \lim _{n \rightarrow \infty} \frac{\log Z_{G_{n}}\left(z_{n}\right)}{\log \left(L\left(z_{n}, G_{n}\right)+1\right)}+\frac{\left(x_{n}-z_{n}\right) \cdot L\left(z_{n}, G_{n}\right)}{z_{n} \cdot \log \left(L\left(z_{n}, G_{n}\right)+1\right)} \\
& \geq 1+\frac{\varepsilon}{2(1+\varepsilon)} \cdot \lim _{n \rightarrow \infty} \frac{L\left(z_{n}, G_{n}\right)}{\log \left(L\left(z_{n}, G_{n}\right)+1\right)}=\infty
\end{aligned}
$$

\section{Acknowledgements}

Thanks to Vincent Beffara, Itai Benjamini and Hugo Duminil-Copin for useful discussions. Thanks to the anonymous referees for their useful remarks.

\section{References}

S. E. Alm and S. Janson. Random self-avoiding walks on one-dimensional lattices. Comm. Statist. Stochastic Models 6 (2), 169-212 (1990). MR1047969.

N. Alon, I. Benjamini, E. Lubetzky and S. Sodin. Non-backtracking random walks mix faster. Commun. Contemp. Math. 9 (4), 585-603 (2007). MR2348845.

R. Bauerschmidt, H. Duminil-Copin, J. Goodman and G. Slade. Lectures on selfavoiding walks. In Probability and statistical physics in two and more dimensions, volume 15 of Clay Math. Proc., pages 395-467. Amer. Math. Soc., Providence, RI (2012). MR3025395. 
D. Brydges, A. Dahlqvist and G. Slade. The strong interaction limit of continuoustime weakly self-avoiding walk. In Probability in complex physical systems, volume 11 of Springer Proc. Math., pages 275-287. Springer, Heidelberg (2012). MR3372852.

D. Brydges, S. N. Evans and J. Imbrie. Self-avoiding walk on a hierarchical lattice in four dimensions. Ann. Probab. 20 (1), 82-124 (1992). MR1143413.

D. Brydges and J. Imbrie. End-to-end distance from the Green's function for a hierarchical self-avoiding walk in four dimensions. Comm. Math. Phys. 239 (3), 523-547 (2003a). MR2000928.

D. Brydges and J. Imbrie. Green's function for a hierarchical self-avoiding walk in four dimensions. Comm. Math. Phys. 239 (3), 549-584 (2003b). MR2000929.

D. Brydges, J. Imbrie and G. Slade. Functional integral representations for selfavoiding walk. Probab. Surv. 6, 34-61 (2009). MR2525670.

D. Brydges and G. Slade. Renormalisation group analysis of weakly self-avoiding walk in dimensions four and higher. In Proceedings of the International Congress of Mathematicians. Volume IV, pages 2232-2257. Hindustan Book Agency, New Delhi (2010). MR2827969.

D. Brydges and T. Spencer. Self-avoiding walk in 5 or more dimensions. Comm. Math. Phys. 97 (1-2), 125-148 (1985). MR782962.

H. Duminil-Copin and A. Hammond. Self-avoiding walk is sub-ballistic. Comm. Math. Phys. 324 (2), 401-423 (2013). MR3117515.

H. Duminil-Copin, G. Kozma and A. Yadin. Supercritical self-avoiding walks are space-filling. Ann. Inst. Henri Poincaré Probab. Stat. 50 (2), 315-326 (2014). MR3189073.

R. Fitzner. Non-backtracking lace expansion: the NoBLE project. Ph.D. thesis, Technische Universiteit Eindhoven (2013).

G. R. Grimmett and Z. Li. Counting self-avoiding walks. ArXiv Mathematics e-prints (2013). arXiv: 1304.7216.

T. Hara. Decay of correlations in nearest-neighbor self-avoiding walk, percolation, lattice trees and animals. Ann. Probab. 36 (2), 530-593 (2008). MR2393990.

T. Hara and G. Slade. Critical behaviour of self-avoiding walk in five or more dimensions. Bull. Amer. Math. Soc. (N.S.) 25 (2), 417-423 (1991). MR1093059.

T. Hara and G. Slade. The lace expansion for self-avoiding walk in five or more dimensions. Rev. Math. Phys. 4 (2), 235-327 (1992a). MR1174248.

T. Hara and G. Slade. Self-avoiding walk in five or more dimensions. I. The critical behaviour. Comm. Math. Phys. 147 (1), 101-136 (1992b). MR1171762.

D. Ioffe. Ornstein-Zernike behaviour and analyticity of shapes for self-avoiding walks on $\mathbf{Z}^{d}$. Markov Process. Related Fields 4 (3), 323-350 (1998). MR1670027.

H. Kesten. On the number of self-avoiding walks. II. J. Mathematical Phys. 5, 1128-1137 (1964). MR0166845.

G. F. Lawler, O. Schramm and W. Werner. On the scaling limit of planar selfavoiding walk. In Fractal geometry and applications: a jubilee of Benoît Mandelbrot, Part 2, volume 72 of Proc. Sympos. Pure Math., pages 339-364. Amer. Math. Soc., Providence, RI (2004). MR2112127.

A. Lubotzky, R. Phillips and P. Sarnak. Ramanujan graphs. Combinatorica 8 (3), 261-277 (1988). MR963118. 
N. Madras and G. Slade. The self-avoiding walk. Modern Birkhäuser Classics. Birkhäuser/Springer, New York (2013). ISBN 978-1-4614-6024-4; 978-1-46146025-1. Reprint of the 1993 original. MR2986656.

N. Madras and C. C. Wu. Self-avoiding walks on hyperbolic graphs. Combin. Probab. Comput. 14 (4), 523-548 (2005). MR2160417.

G. A. Margulis. Explicit constructions of graphs without short cycles and low density codes. Combinatorica 2 (1), 71-78 (1982). MR671147.

A. Nachmias. Mean-field conditions for percolation on finite graphs. Geom. Funct. Anal. 19 (4), 1171-1194 (2009). MR2570320.

A. Nachmias and Y. Peres. Non-amenable Cayley graphs of high girth have $p_{c}<p_{u}$ and mean-field exponents. Electron. Commun. Probab. 17, no. 57, 8 (2012). MR3005730. 